SHEP 92/93-22

\title{
Low Scale Technicolour at LEP
}

\author{
S. F. King, \\ Physics Department, \\ University of Southampton, \\ Southampton, \\ SO9 $5 \mathrm{NH}$, \\ $U . K$.
}

\begin{abstract}
We discuss the phenomenology of an $S U(2)_{T C}$ technicolour model with a low technicolour confinement scale $\Lambda_{T C} \sim 50-100 \mathrm{GeV}$. Such a low technicolour scale may give rise to the first hints of technicolour being seen at LEPI and spectacular technicolour signals at LEPII.
\end{abstract}


Recently we proposed a dynamical model of leptons in which the leptons were endowed with three colours (red, white and blue) where two of the colours (red and blue) split off to form a technicolour (TC) group $S U(2)_{T C}$, while the third colour (white) is identified with the physical leptons [1]. Fermion masses were generated by fourth family condensates [1]. In this paper we discuss the phenomenology of a three family version of this model on the assumption that the TC group confines at a low technicolour confinement 円 scale $\Lambda_{T C} \sim 50-100 \mathrm{GeV}$, which is a natural assumption in this model. Such a low technicolour scale may give rise to the first hints of technicolour being seen at LEPI and spectacular technicolour signals at LEPII.

When the three lepton colours have split apart as discussed above, the model corresponds to the moose diagram in Fig.1. The exchange of the various heavy gauge bosons which mix together via loops of preons gives rise to contact operators in the effective low energy theory which describes the three families of leptons and technifermions. The key features of this model relevant to our phenomenological discussion are listed below:

- Technicolour $S U(2)_{T C}$ confines at $\Lambda_{T C} \sim 50-100 \mathrm{GeV}$.

- There are three technidoublets closely associated with three lepton families, but only coupling to them weakly via chirality conserving operators.

- One dominant technidoublet $T$ (associated with the $\tau$ family) is mainly responsible for electroweak symmetry breaking and has a heavy dynamical mass on the order of $500-1000 \mathrm{GeV}$.

- The remaining two technidoublets $t_{1}, t_{2}$ (associated with the $e$ and $\mu$ families) have light dynamical masses on the order of $\Lambda_{T C} \sim 50-100 \mathrm{GeV}$.

\footnotetext{
${ }^{1}$ Our definition of the confinement scale is that it is equal to one half of the mass of the lowest lying vector resonance.
} 
The following discussion will apply to any TC model which has the above features, and not just the model in Fig.1.

To begin with let us consider the dominant technidoublet $T=(P, M)$ 2. In this model the exchange of heavy flavour gauge bosons induces operators of the form [1] $G_{T}\left(\bar{T}_{L} T_{R}\right)\left(\bar{T}_{R} T_{L}\right)$. There are similar contact terms of the form $G_{\tau}\left(\bar{\tau}_{L} \tau_{R}\right)\left(\bar{\tau}_{R} \tau_{L}\right)$ which induce a tau lepton condensate [1] and other contact terms such as $G_{\tau \mu}\left(\bar{\tau}_{L} \tau_{R}\right)\left(\bar{\mu}_{R} \mu_{L}\right)$ allow the tau mass to be fed down to the muon (and similarly for the electron). Other contact terms such as $G_{\mu}\left(\bar{\mu}_{L} \mu_{R}\right)\left(\bar{\mu}_{R} \mu_{L}\right)$ have smaller coefficients. Quark masses are generated by top and bottom quark condensates [3, 4]. Since we assume a tau condensate then $G_{T} \approx G_{\tau}$ must therefore be strong, leading to a condensate $<\bar{P} P+$ $\bar{M} M>\neq 0$. If the operator respects isospin then the pattern of symmetry breaking f expected is just

$$
S U(2)_{L} \otimes S U(2)_{R} \rightarrow S U(2)_{L+R}
$$

yielding a triplet of technipions $\Pi_{T C}^{ \pm, 0} \sim \bar{T} \sigma^{ \pm, 3} \gamma_{5} T$, where $\sigma^{a}$ are the Pauli matrices. In such a theory the technipion decay constant may be much larger than $\Lambda_{T C}$ since chiral symmetry breaking is driven mainly by the above contact operator [5]. Thus we may have, $<0\left|J_{\mu 5}^{a}\right| \Pi_{T C}^{b}>=i F_{T C} q_{\mu} \delta^{a b}$, where $F_{T C} \sim 245 G e V$, and the current is $J_{\mu 5}^{a}=\bar{T} \gamma_{\mu} \gamma_{5} \sigma^{a} T$. All these technipions get eaten, and the remaining technihadrons have masses set by the enhanced dynamical masses of the technifermions, and are in the $\mathrm{LHC} / \mathrm{SSC}$ range.

Now let us extend our discussion to include one of the other technidoublets $t_{2}$ in this model, where $t_{2}$ is associated with the muon family. The technidoublet $t_{1}$

\footnotetext{
${ }^{2}$ Each techniquark carries TC but not ordinary colour, and the left-handed techniquarks $T_{L}$ form an $S U(2)_{L}$ doublet just like ordinary quarks but with weak hypercharge $Y=0$. The righthanded techniquarks $P_{R}, M_{R}$ have $Y=1 / 2,-1 / 2$, respectively. The electric charge generator is given by $Q=T_{L 3}+Y$ so that the plus $(P)$ and minus $(M)$ techniquarks have charges given by $Q= \pm 1 / 2$,respectively.

${ }^{3}$ The full $S U(4)$ symmetry of a one-doublet $S U(2)_{T C}$ model is broken here by the fourtechnifermion operators. We assume these operators respect isospin symmetry for convenience only; in reality there will be some isospin violation.
} 
will be considered later. There are now two technidoublets $T=(P, M), t=(p, m)$, which have identical quantum numbers (we have dropped the subscript on $t_{2}$ for convenience). The set of operators in this case are of the form [1]

$$
G_{T}\left(\bar{T}_{L} T_{R}\right)\left(\bar{T}_{R} T_{L}\right), G_{t}\left(\bar{t}_{L} t_{R}\right)\left(\bar{t}_{R} t_{L}\right), G_{t T}\left(\bar{T}_{L} T_{R}\right)\left(\bar{t}_{R} t_{L}\right)
$$

where $G_{t}, G_{t T} \ll G_{T}$ since $G_{t} \approx G_{\mu}$ and $G_{t T} \approx G_{\tau \mu}$. In this case the theory naturally splits into two parts, a high scale TC sector associated with the technifermions $T$ which form condensates driven by the operators associated with the scale $\Lambda \sim 1 T e V$, as discussed above, and a low scale TC sector associated with technifermions $t$ which form condensates driven by TC interactions at the scale $\Lambda_{T C} \sim 50-100 \mathrm{GeV}$. The low scale TC sector again has an approximate global symmetry as in Eq.(1), but now there is a vacuum alignment problem which depends on the relative strength of the contact term and the TC gauge forces. The TC gauge forces tend to favour the chiraly invariant condensate $<\bar{t}_{L}^{\bar{c}} t_{L}+\bar{t}_{R}^{\bar{c}} t_{R}>\neq 0$, while the contact terms prefer the chiral symmetry breaking condensates, $\left\langle\overline{t_{L}} t_{R}+\overline{t_{R}} t_{L}>\neq 0\right.$. We shall return to this point later. For now we shall assume the latter condensates form, yielding a triplet of technipions $\pi_{T C}^{ \pm, 0} \sim \bar{t} \sigma^{ \pm, 3} \gamma_{5} t$, with $<0\left|j_{\mu 5}^{a}\right| \pi_{T C}^{b}>=i f_{T C} q_{\mu} \delta^{a b}$, where $f_{T C} \sim$ $10-25 \mathrm{GeV}$, and the current is $j_{\mu 5}^{a}=\bar{t} \gamma_{\mu} \gamma_{5} \sigma^{a} t$. There are two points of interest concerning the low scale TC sector. Firstly the low scale technipion triplet do not get eaten. I More importantly the low scale TC sector may be accessible to existing colliders such as LEP, as we now discuss.

The physical technipions $\pi_{T C}^{ \pm, 0}$ will receive a mass from the mixed operator with coefficient $G_{t T}$ in Eq.(2). The "explicit" masses of the low scale technifermions resulting from this operator are obviously just $m_{p, m}=G_{t T}\left\langle\left(\bar{T}_{L} T_{R}\right)\right\rangle$, which may be estimated since $G_{t T} \approx G_{\tau \mu}$. These masses break the chiral symmetry of the second

\footnotetext{
${ }^{4}$ Strictly the eaten and physical technipions are given by leaten technipion $>=$ $\frac{F_{T C}\left|\Pi_{T C}^{ \pm, 0}>+f_{T C}\right| \pi_{T C}^{ \pm, 0}>}{\sqrt{F_{T C}^{2}+f_{T C}^{2}}}$ and $\mid$ physical technipion $>=\frac{f_{T C}\left|\Pi_{T C}^{ \pm, 0}>-F_{T C}\right| \pi_{T C}^{ \pm, 0}>}{\sqrt{F_{T C}^{2}+f_{T C}^{2}}}$. The full current is $J_{5 \mu}+j_{5 \mu}$, where, $<0\left|J_{5 \mu}+j_{5 \mu}\right| \Pi_{T C}^{ \pm, 0}>=F_{T C} q^{\mu}$ and $<0\left|J_{5 \mu}+j_{5 \mu}\right| \pi_{T C}^{ \pm, 0}>=f_{T C} q^{\mu}$, which implies, $<0\left|J_{5 \mu}+j_{5 \mu}\right|$ eaten technipion $>=\sqrt{F_{T C}^{2}+f_{T C}^{2}} q^{\mu}$ and $<0\left|J_{5 \mu}+j_{5 \mu}\right|$ physical technipion $>=0$.
} 
technidoublet resulting in a physical technipion mass analagous to the way in which the physical pion mass results from explicit quark masses. The technipion mass $m_{\pi_{T C}}$ may be estimated by scaling up the usual result for the ordinary pion mass $m_{\pi}$,

$$
m_{\pi_{T C}}=m_{\pi} \sqrt{\left(\frac{m_{p}+m_{m}}{m_{u}+m_{d}}\right) \frac{f_{T C}}{f_{\pi}}} .
$$

Using Eq.(3) combined with our estimate of $m_{p, m}$ we find rather heavy technipion masses of order tens of GeV. LEPI sets a limit on the mass of charged technipion masses of around $M_{Z} / 2$.

Assuming the technifermions have very small couplings to leptons (the chirality conserving couplings are discussed later) the charged technipions $\pi_{T C}^{ \pm}$will decay predominantly via virtual $\mathrm{W}$ exchange, analagous to ordinary charged pion decay. Thus for example the width into leptons is given by,

$$
\Gamma\left(\pi_{T C}^{ \pm} \rightarrow l^{ \pm} \nu_{l}\right)=\frac{f_{T C}^{2}}{f_{\pi}^{2}} \frac{m_{l}^{2}}{m_{\mu}^{2}} \frac{m_{\pi_{T C}}}{m_{\pi}} \frac{1}{\left(1-\frac{m_{\mu}^{2}}{m_{\pi}^{2}}\right)^{2}} \Gamma\left(\pi^{ \pm} \rightarrow \mu^{ \pm} \nu_{\mu}\right)
$$

The largest such decay channels are thus $b c, c s, \tau \nu_{\tau}$. The neutral technipions $\pi_{T C}^{0}$ may also decay by this mechanism, via virtual $\mathrm{Z}$ exchange, with the largest partial width,

$$
\Gamma\left(\pi_{T C}^{0} \rightarrow \bar{b} b\right) \approx 3\left(\frac{m_{b}^{2}}{m_{l}^{2}}\right) \Gamma\left(\pi_{T C}^{ \pm} \rightarrow l^{ \pm} \nu_{l}\right)
$$

$\pi_{T C}^{0}$ will also decay into two photons via a chiral symmetry suppressed anomalous $\pi_{T C}^{0} \gamma \gamma$ coupling. The $\pi_{T C}^{0} \gamma \gamma$ coupling proportional to

$$
S_{\pi_{T C}^{0} \gamma \gamma}=N_{T C} \operatorname{Tr}\left[\frac{\sigma^{3}}{2}\left(\left\{\Gamma_{V}, \Gamma_{V}\right\}+\left\{\Gamma_{A}, \Gamma_{A}\right\}\right) \frac{\mu M}{\Lambda_{T C}^{2}}\right]
$$

where $\Gamma_{A}=0, \Gamma_{V}=Q=e \frac{\sigma_{3}}{2}, M=\operatorname{diag}\left(m_{p}, m_{m}\right)$ is the technifermion mass matrix, and $\operatorname{Tr} \mu M \approx m_{\pi_{T C}}^{2}$. The usual anomalous coupling without the mass factor is zero in this model. Providing that there is some isospin violation (i.e. $m_{p} \neq m_{m}$ ) the contribution to this coupling will be non-zero, and will be only mildly suppressed relative to the usual anomalous coupling by a factor of roughly $m_{\pi_{T C}}^{2} / \Lambda_{T C}^{2}$. The 
partial width is given by [6],

$$
\Gamma\left(\pi_{T C}^{0} \rightarrow \gamma \gamma\right)=A_{\pi_{T C}^{0} \gamma \gamma}^{2}\left(\frac{\alpha^{2}}{16 \pi^{3} f_{T C}^{2}}\right) m_{\pi_{T C}^{0}}^{3}
$$

where $S_{\pi_{T C}^{0} \gamma \gamma}=A_{\pi_{T C}^{0} \gamma \gamma} 2 e^{2}$, where in the present model $A_{\pi_{T C}^{0} \gamma \gamma}=-(1 / 8)\left(\frac{m_{\pi_{T C}^{0}}}{\Lambda_{T C}}\right)^{2}$. Despite its small partial width, this will be an important decay mode of the $\pi_{T C}^{0}$.

Apart from the low-scale technipions, the technidoublet $t$ will give rise to technivector mesons $V$ analagous to the QCD vector resonances. However here the masses of such technivectors will be an order of magnitude smaller than in conventional TC. For example we may expect a $J^{P C}=1^{--}$technirho triplet $\rho_{T C}^{ \pm, 0}$ and techniomega singlet $\omega_{T C}$ with masses in the LEPII range $100-200 \mathrm{GeV}$. The vector masses may be estimated by scaling up the ordinary $\rho$ and $\omega$ mass $m_{\rho_{T C}, \omega_{T C}} \approx m_{\rho, \omega} \frac{f_{T C}}{f_{\pi}}$. The technidoublet $t$ has photon and $\mathrm{Z}$ couplings,

$$
A_{\mu} \bar{t} \gamma^{\mu} Q t+Z_{\mu} \bar{t} \gamma^{\mu}\left(\Gamma_{V}+\Gamma_{A} \gamma_{5}\right) t
$$

where

$$
Q=e \frac{\sigma^{3}}{2}, \quad \Gamma_{V}=\frac{e}{\tan 2 \theta_{w}} \frac{\sigma^{3}}{2}, \quad \Gamma_{A}=\frac{-e}{\sin 2 \theta_{w}} \frac{\sigma^{3}}{2} .
$$

Using vector meson dominance arguments, combined with scaling-up arguments, we write,

$$
\bar{t} \gamma^{\mu} \frac{\sigma^{a}}{2} t \rightarrow \frac{m_{\rho_{T C}}^{2}}{g_{\rho_{T C}}} \rho_{T C}^{a \mu}
$$

where $g_{\rho_{T C}} \approx g_{\rho}$, and $g_{\rho}=\sqrt{12 \pi}$. Thus the $\rho_{T C}^{0}$ may be detected at LEPII via its couplings to the photon and Z,

$$
\frac{m_{\rho_{T C}}^{2}}{g_{\rho_{T C}}} \rho_{T C}^{0, \mu}\left[e A_{\mu}+\frac{e}{\tan 2 \theta_{w}} Z_{\mu}\right]
$$

leading to resonances in $\mathrm{R}$. The techniomega $\omega_{T C}^{0}$ being associated with the isosinglet current $\bar{t} \gamma^{\mu} t$ does not couple to the photon or Z.

If the technirho mass is above two technipion threshold then pairs of technipions may be produced at resonantly enhanced rates. The partial width of the technirho 
into a pair of charged technipions is given by a scaling argument as,

$$
\Gamma\left(\rho_{T C} \rightarrow \pi_{T C}^{+} \pi_{T C}^{-}\right)=\frac{m_{\rho_{T C}}}{m_{\rho}} \frac{\beta_{\pi_{T C}}^{3}}{\beta_{\pi}^{3}} \Gamma\left(\rho \rightarrow \pi^{+} \pi^{-}\right)
$$

where $\beta_{\pi(T C)}=\left(1-\frac{4 m_{\pi(T C)}^{2}}{m_{\rho(T C)}^{2}}\right)^{1 / 2}$. The technivector mesons may also have direct couplings to leptons via four-fermion operators which couple technifermions to leptons but do not violate chirality, which arise from exchange of the flavour diagonal gauge bosons. Neutrino couplings may be controlled independently in this model. In addition $t=(p, m)$ (where $m$ is effectively a coloured muon) will have independent couplings to muons arising from heavy lepton colour gauge bosons which convert a lepton into a technicoloured lepton. Thus direct techniomega coupling to leptons may originate from four-fermion operators of the form $H_{l} \bar{t} \gamma_{\mu} t \bar{l} \gamma_{\mu} l$ where $l=e, \mu, \tau, \nu_{e}, \nu_{\mu}, \nu_{\tau}$. The relative strengths of each of the neutrino and muon couplings can be adjusted in this model. The operator induces a direct lepton coupling @,

$$
H_{l} \frac{m_{\omega_{T C}}^{2}}{g_{\omega_{T C}}} \omega_{T C}^{\mu} \bar{l} \gamma_{\mu} l .
$$

Recently the L3 collaboration at LEP [7] reported four events, one $e^{+} e^{-} \gamma \gamma$ and three $\mu^{+} \mu^{-} \gamma \gamma$, each with two energetic photons with an invariant mass $M_{\gamma \gamma} \approx 60 \mathrm{GeV}$. Other LEP groups have informally reported one or two similar events, plus one or two $\bar{\nu} \nu \gamma \gamma$ and $\bar{q} q \gamma \gamma$ events but such events appear to be consistent with background. Such events are also consistent with a $60 \mathrm{GeV}$ neutral technipion, as we now discuss. From Eq.(7) we find $\Gamma\left(\pi_{T C}^{0} \rightarrow \gamma \gamma\right)=10 \mathrm{keV}$ (assuming $\left.\Lambda_{T C}=50 \mathrm{GeV}, f_{T C}=10 \mathrm{GeV}\right)$. Similarly from Eqs. $(4,5)$ we find $\Gamma\left(\pi_{T C}^{0} \rightarrow \bar{b} b\right)=4 k e V$. The two photon decay is probably too narrow to enable the technipion to be produced by two photon collisions at LEP. The partial width of the technipion into $e^{+} e^{-}$is heavily suppressed compared to its width into $\bar{b} b$, and is well below the sensitivity of the recent TRISTAN search 8], which is sensitive to electronic widths of a few $\mathrm{keV}$.

\footnotetext{
${ }^{5}$ The same operator will also induce direct couplings of $\pi_{T C}^{0}$ to leptons of the form $H_{l} f_{T C} \partial_{\mu} \pi_{T C}^{0} \bar{l} \gamma_{\mu} l$.
} 
Now let us consider the production rate of a $60 \mathrm{GeV}$ technipion at LEP. The conventional rate [6] for $Z \rightarrow \pi_{T C}^{0} l^{+} l^{-}$via a virtual $Z$ is much too small to be relevant at LEP. However since the technivector mesons in this model are light then there will be an additional production mechanism for the technipions via a $Z V \pi_{T C}^{0}$ vertex where $\mathrm{V}$ is a technivector meson, either a technirho or a techniomega. This coupling is much stronger than typical anomalous couplings since it involves two strong and one electroweak couplings, rather than two electroweak and one strong coupling. This coupling combined with Eq.(13) will allow the decay 0

$$
Z \rightarrow \pi_{T C}^{0}+\omega_{T C}^{*}, \omega_{T C}^{*} \rightarrow \overline{l l}
$$

The precise rate will depend on the $\omega_{T C}$ mass, and the strength of the $Z \omega_{T C} \pi_{T C}^{0}$ vertex, and is difficult to estimate in this model. The decay rate may be approximately scaled on the standard model Higgs h production rate via a virtual Z,

$$
\frac{\Gamma\left(Z \rightarrow \pi_{T C}^{0} \bar{l} l\right)}{\Gamma(Z \rightarrow h \bar{l} l)} \approx R_{Z \omega_{T C} \pi_{T C}^{0}}^{2} R_{\omega_{T C} \bar{l} l}^{2} \frac{M_{Z}^{4}}{m_{\omega_{T C}}^{4}}
$$

where we have taken ratios of couplings to those in the standard model process. There is no reason why these $R$ ratios should not be of order unity, and, since the $\omega_{T C}$ mass is light in this model, it appears to be possible to account for the $60 \mathrm{GeV}$ two photons events, accompanied by pairs of electrons, muons, taus or neutrinos in adjustable ratios. The technipion may also be produced in association with a virtual technirho which couples to $\bar{q} q$ jets via its photon or Z couplings in Eq.(11).

A low mass techniomega will contribute to Bhabha scattering due to the direct coupling to leptons in Eq.(13), which will generate an effective four-electron contact interaction. According to current limits on the coefficient of such contact interactions we require

$$
\frac{\alpha_{\omega_{T C} e e}}{m_{\omega_{T C}}^{2}}<\frac{4 \pi^{2}}{(1 T e V)^{2}}
$$

\footnotetext{
${ }^{6} \mathrm{~A}$ similar mechanism was proposed by Bando and Maekawa[9], except that these authors assumed that the real on-shell $\mathrm{Z}$ will decay into a real on-shell $\pi_{T C}^{0}$ plus a virtual scalar technihadron.
} 
where $\alpha_{\omega_{T C} e e}=\left(H_{e} \frac{m_{\omega_{T C}}^{2}}{g_{\omega_{T C}}}\right)^{2}$ from Eq.(13). Clearly if $\alpha_{\omega_{T C} e e}$ is of electroweak strength and the techniomega mass is as low as $100 \mathrm{GeV}$, as assumed above, then this bound is close to being realised. Thus at LEPII one may hope to use Bhabha scattering as a probe of the techniomega, and perhaps even produce an on-shell techniomega via the direct lepton coupling in Eq.(13).

In the above discussion we considered only $t_{2}$ (which we wrote as $t$ ). Now we must consider the technidoublet $t_{1}$ which is associated with the electron family. In this case it will be necessary to assume that $t_{1}$ forms electroweak preserving condensates since otherwise the tau lepton mass will be fed down to the electron via flavour operators generated by diagrams involving internal technifermion condensates. Operators of the form $K_{L}\left(\bar{e}_{L} \gamma_{\nu} \tau_{L}\right)\left(\bar{m}_{1 L} \gamma^{\nu} M_{L}\right)$ and $K_{R}\left(\bar{e}_{R} \gamma_{\nu} \tau_{R}\right)\left(\bar{m}_{1 R} \gamma^{\nu} M_{R}\right)$ may be fused together via technifermion condensates to form operators which (after a Fierz transformation) have the form $J\left({\overline{e_{L}}}_{R}\right)\left(\overline{\tau_{L}} \tau_{R}\right)$. This operator leads to an electron mass of order $\left(\frac{\Lambda_{T C}}{\Lambda}\right) m_{\tau}$, which is too large assuming $\left(\frac{\Lambda_{T C}}{\Lambda}\right) \approx 0.1$. Such a mass is barely acceptable for the muon. But for the electron we must prevent such operators from being constructed and the simplest way to do this is by assuming that $<\bar{m}_{1 L} m_{1 R}>=0$. Instead we assume the invariant condensates $<p_{1 L}^{\bar{c}} m_{1 L}-\bar{m}_{1 L}^{c} p_{1 L}+L \rightarrow R>\neq 0$. It is natural to assume that $t_{1}$ forms invariant condensates but $t_{2}$ forms chiral symmetry breaking condensates since the contact operators which prefer the broken condensates are weaker for the electron than for the muon.

It is possible that $t_{1}, t_{2}$ both form chirally invariant condensates so that all the technihadrons will have masses $\approx 2 \Lambda_{T C}$. In this case it is possible that the tau lepton mass is generated not from tau lepton condensates but is fed down from the TC sector via exchange of the heavy gauge bosons which unify the three lepton colours [1]. The contact operators of interest are, after a Fierz transformation,

$$
K\left[\left(\bar{\tau}_{L} \tau_{R}\right)\left(\bar{M}_{R} M_{L}\right)+\left(\bar{\mu}_{L} \mu_{R}\right)\left(\bar{m}_{2 R} m_{2 L}\right)+\left(\bar{e}_{L} e_{R}\right)\left(\bar{m}_{1 R} m_{1 L}\right)\right]
$$


The tau mass from this mechanism is $m_{\tau}=K<\bar{M}_{R} M_{L}>$, where $\mathrm{K}$ is proportional to an explicit preon mass. The $e$ and $\mu$ receive no mass from these operators having assumed invariant condensates for $t_{1}, t_{2}$, but their mass is fed down from the tau lepton in the usual way. The smallness the tau mass implies that the chirality breaking operators in Eq.(17) above are phenomenologically unimportant.

The above mechanism avoids the fine-tuning associated with tau condensates but there will still be some fine tuning required in the quark sector since we require an independent $\mathrm{b}$ quark condensate. All fine tuning can be eliminated by extending this scenario to include fourth family condensates [1]. In this case there will be an electroweak breaking technidoublet $T$ associated with the fourth lepton family, but it will be necessary to assume that all three low scale technidoublets $t_{1}, t_{2}, t_{3}$ (where $t_{3}$ is now associated with the tau family) form electroweak preserving condensates in order to prevent too large a tau mass arising from the operators discussed above. In such a fourth family variant of this model there may be chirality violating couplings as in Eq.(17) controlled by a coupling $\mathrm{K}$ which may be much larger than in the three family scenario. Such couplings will allow spin zero bound states of mass $\sim 2 \Lambda_{T C}$ to decay to electrons or muons at rates which are unsuppressed by the lepton mass. Such spin zero states may be resonantly produced in $e^{+} e^{-}$collisions.

Finally we must consider the constraints on this model from high precision tests of the standard model. At LEPI energies, assuming the $\rho_{T C}$ mass for example is much greater than $M_{Z}$ (a somewhat questionable assumption in this model) the technirho will contribute to the oblique corrections to the photon and $\mathrm{Z}$ propagators. For example the contribution to the S parameter [10] for a single technidoublet is estimated to be $S \approx 0.3\left(\frac{2}{3}\right) \approx 0.2$. This estimate is based on vector meson dominance and large $N_{T C}$ scaling arguments in order to estimate the contribution to dispersion relations. The contribution depends on scale independent ratios like $\frac{f_{T C}}{m_{\rho_{T C}}}$ so is independent of the fact that the technicolour scale (and the technirho mass) is low. The dom- 
inant technidoublet $T$ may contribute somewhat differently since its dynamics are not QCD-like, but are controlled by the contact operator. The technidoublet $t_{1}$ also forms non QCD-like condensates. All together from the three technidoublets $T, t_{1}, t_{2}$ we might expect a contribution to the S parameter of order 0.6 in this model, subject to the uncertainties mentioned above. In the four family scenario there will of course be additional contributions.

In conclusion, we have discussed the LEP phenomenology of a technicolour model with a low confinement scale. The specific model is summarised in Fig.1, but our discussion will apply to any pure TC (i.e. unextended TC) model which has the listed features. As we have seen such models offer the prospect of exciting physics at LEPII with perhaps the first signs of new physics visible at LEPI. The high scale TC sector may be studied at LHC or SSC in the usual way. This really is a jam today jam tomorrow scenario!

\section{Acknowledgements}

SFK would like to thank Nicholas Evans, Jonathan Flynn, Lisa Randall, Douglas Ross and Terry Sloan for discussions and the SERC for financial support. 


\section{Figure Caption}

Figure 1

A three family lepton moose model. As usual, the circles represent gauge groups and the lines represent fermions which transform under the gauge groups. The labelled lines in the lower half of the diagram correspond to three families of leptons (including right-handed neutrinos) denoted $(N, E)_{L}, N_{R}^{c}, E_{R}^{c}$ and three families of technifermions, denoted $(P, M)_{L}, P_{R}^{c}, M_{R}^{c}$. The three families of leptons and technifermions share acommon gauge chiral family symmetry $S U(3)_{L} \otimes S U(3)_{E} \otimes S U(3)_{N}$. The remaining lines represent preons which transform under the preon gauge groups $S U(3)_{f E}$ and $S U(3)_{f N}$ which confine at $\Lambda \sim 1 T e V$. The preons in the upper half of the diagram have explicit mass matrices, and condense with the preons represented by horizontal lines at the preon confinement scale. The preon dynamics breaks the gauged chiral family symmetries to global chiral family symmetries, which are explicitly broken by the preon masses, thereby ensuring a GIM mechanism [2]. 


\section{References}

[1] S. F. King, Phys. Rev.D46 (1992) 4097.

[2] R. S. Chivukula and H. Georgi, Phys. Lett. B188 (1987) 99; R. S. Chivukula, H. Georgi and L. Randall, Nucl. Phys.B292 (1987) 93.

[3] S. F. King, Phys. Rev.D45 (1992) 990.

[4] T. Elliott and S. F. King, Phys. Lett. B283 (1992) 371.

[5] T. Appelquist, T. Takeuchi, M. Einhorn and L. C. R. Wijewardhana, Phys. Lett. B220 (1989) 223; B. Holdom, Phys. Lett. B226 (1989) 137; S. F. King and D. A. Ross, Phys. Lett. B236 (1990) 327.

[6] V. Lubicz, Rome "La Sapienza" preprint n.925 (January, 1993).

[7] L3 Collaboration, Phys. Lett. B295 (1993) 337.

[8] VENUS Collaboration, Phys. Lett.B302 (1993) 119.

[9] M. Bando and N. Maekawa, KUNS 1174, HE(TH) 92/14, hep-ph/9212317 (December 1992).

[10] M. Peskin and T. Takeuchi, Phys. Rev. Lett. 65 (1990) 964; ibid Phys. Rev. D46 (1992) 381; R. N. Cahn and M Suzuki, Phys. Rev.D44(1991)3641. 